\title{
Application of doxycycline (DOX)-combined bone substitute on vertical bone augmentation on rabbit calvaria
}

\author{
Hamada T, Ikumi R, Kouzuma W, lijima $H$, Kasugai $S$ \\ Oral Implantology and Regenerative Dental Medicine, \\ Tokyo Medical and Dental University
}

For vertical bone augmentation, using autogenous bone graft is still a golden standard. Since harvesting autogenous bone increases patient's morbidity, bone substitutes are increasingly used in implant therapy. As the amount of soft tissues is insufficient to cover the augmented site for vertical augmentation, the risk of infection exists when grafting with bone substitutes. DOX, one of the antibiotics of the tetracycline family, has been reported to promote bone apposition around bone substitute. The aim of this study was to evaluate the DOX-combined deproteinized bovine mineral (DBBM) as a bone substitute for vertical bone augmentation model.

\section{Methods and Materials}

Eight male Japan white rabbits (6 weeks old, 4.6 4.8kg in weight) were used in this study. They were randomly divided into two groups: DBBM alone (Control) and DBBM-DOX (Experimental). Under anesthesia, straight incision was made in the midline of the forehead and periosteum was reflected laterally. Cylindrical-shaped chambers of $3 \mathrm{~mm}$ in height and $5 \mathrm{~mm}$ in inner diameter were fixed with stainless screw at two opposite sites equally apart from the sagittal suture on the Calvaria. Then the cambers were tightly filled either with DBBM alone or DBBM-DOX. In the experimental group, DBBM in one chamber was combined with $1 \mathrm{mg}$ DOX(Fig1). Thereafter, mucoperiosteal flap was closed by suturing periosteum and skin. Two and 4 weeks after the surgery, the animals were sacrificed with overdose of thiopental sodium. Calvaria including chambers and surrounding bone was harvested for Micro CT analysis to evaluate the amount of newly formed bone(Fig.2). Bone mineral content (BMC) and bone mineral density (BMD) were measured(Fig.3,4).
The formation of newly formed bone from the basal bone was more prominent in the experimental group than in the control

group.

High Bone mineral content(BMC) was found in the DBBM-DOX group at 4weeks.

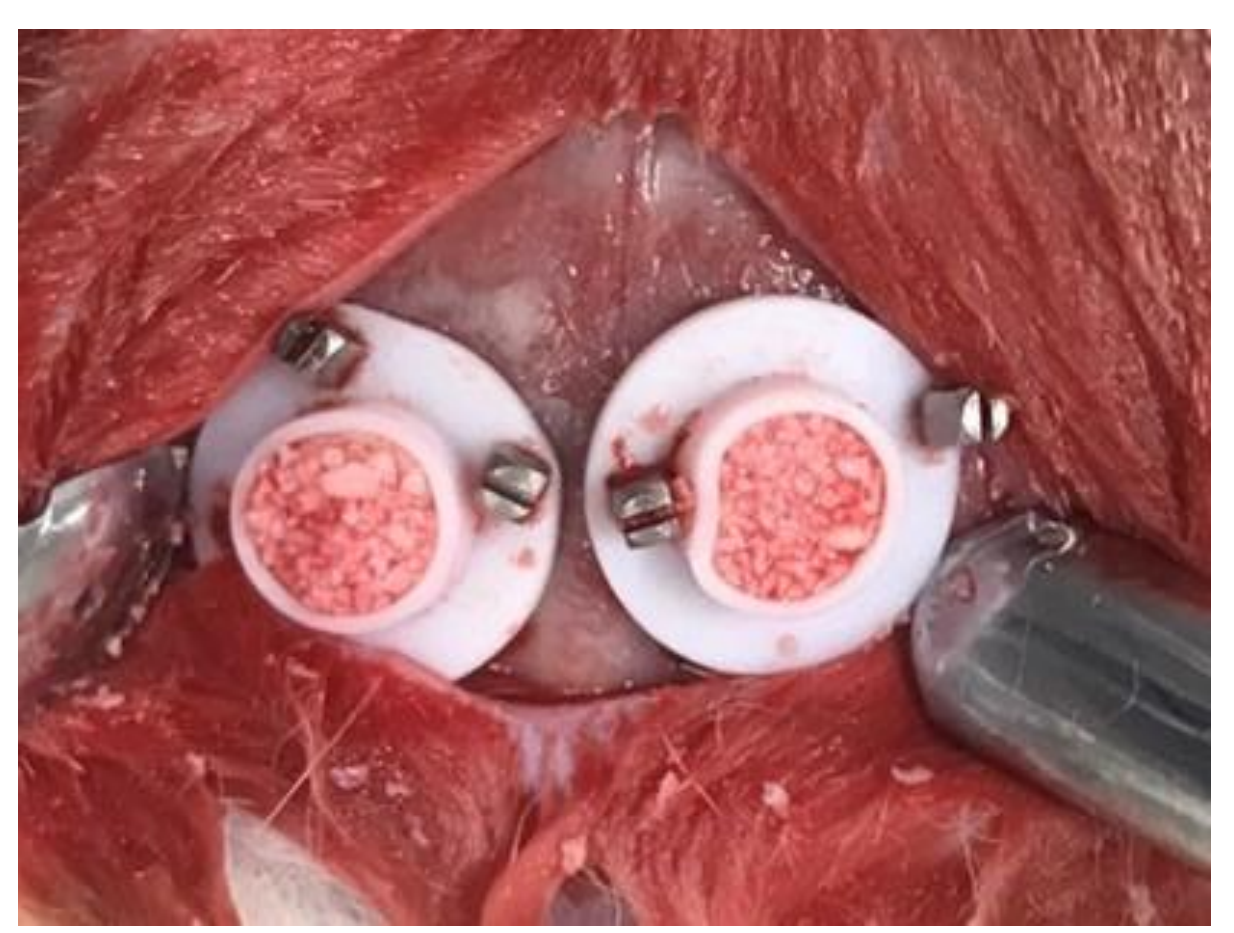

Fig.1

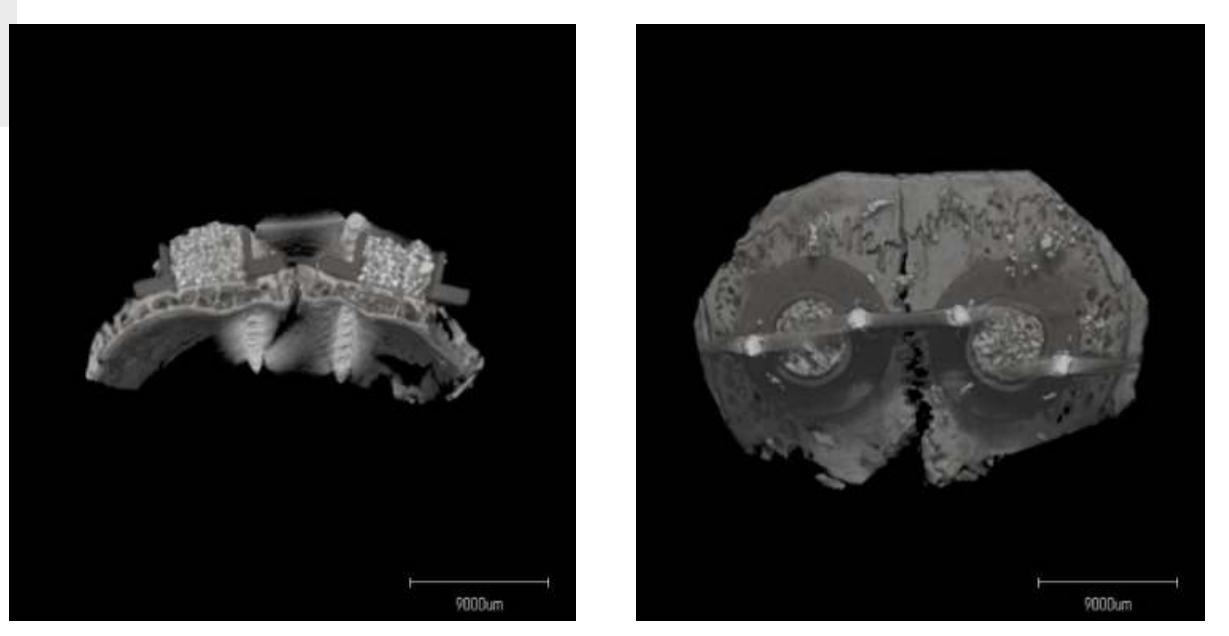

Fig.2

Newly formed bone

$2 \mathrm{~W}$

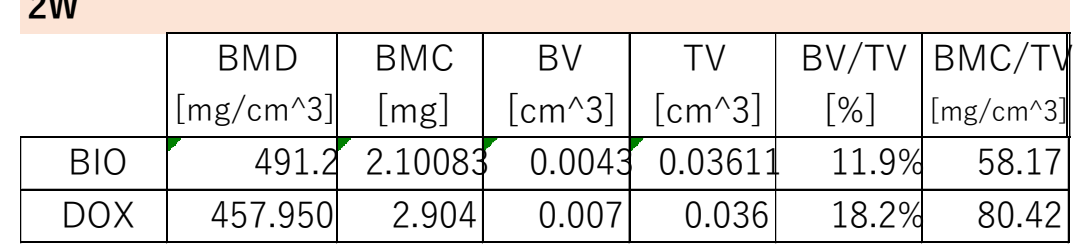

$4 \mathrm{~W}$

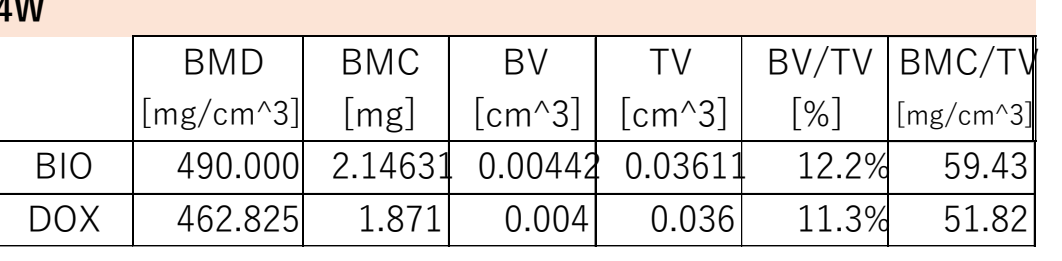

Fig.3

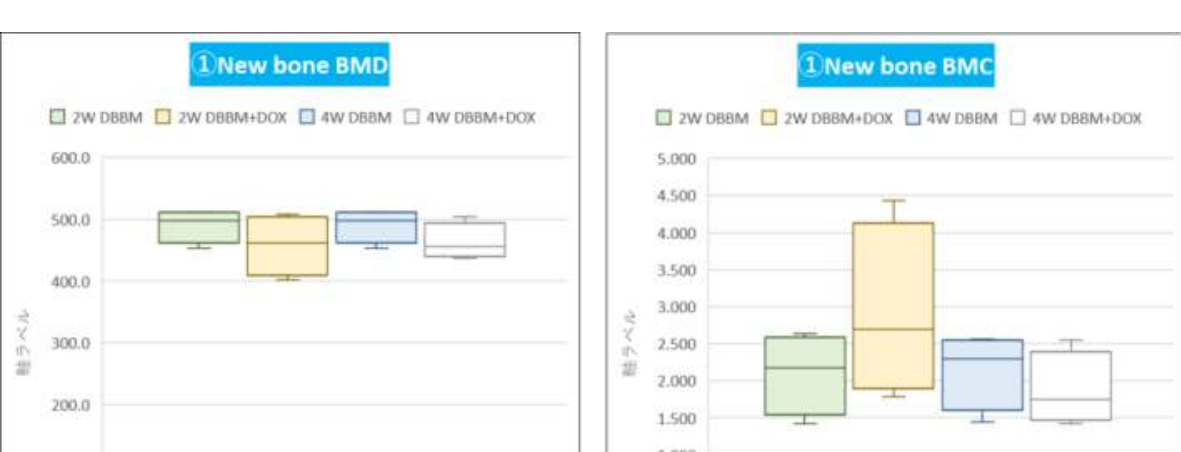

The beneficial effects of the combination of DOX with bone substitutes has been reported previously ${ }^{1}$. We have recently demonstrated that the combination of DOX with DBBM, which was further covered with a collagen membrane in the similar experimental model, stimulated new bone formation compared with DBBM alone ${ }^{2}$. In the present study, the chamber was not covered with collagen membrane.

Nevertheless, new bone formation was still stimulated with DOX combination. Although precise histological analysis is absolutely required in the present study, it likely that a barrier membrane might not be necessary when DBBM is combined with DOX in GBR for vertical augmentation. DOX is an antibiotics of long history and it does not exert severe side effect. In the present study DOX application didn't show any visible adverse effect. Although DOX is not a strong antibiotics, combination with DOX and DBBN can prevent the graft infection, which is a major concern in vertical bone augmentation.

Conclusively, The present results indicate that combination with DOX would be clinically effective when grafting with DBBM for vertical augmentation.

\section{Reference}

\section{Vera Luginbehl,Kurt Ruffieux,Claudia Hess,Dennis Reichardt,Brigitte von Rechenberg,Kajita Nuss}

Controlled Release of Tetracycline from Biodegradable $\beta$-tricalcium Phosphate Composite.Journal of Biomedical Materials Research.92B:341-352,2010

\section{Peng ZHANG, Lin DING,and Shohei KASUGAI}

Effect of doxycycline doped bone substitute on vertical bone augmentation on rat calvaria.Dental Materials Journal 2018

Fig.4 